\title{
Oxidative stress/lipid peroxidation and antioxidant enzymes in apparently healthy individuals involved in physical exercise
}

\author{
Ikekpeazu Joy Ebele', Ikekpeazu Adanna Jennifer'2, Eke Christian Nnabugo ${ }^{3}$, \\ Ogbu Innocent Sidney ${ }^{4}$, Onyekwelu Kenechukwu Chibuike ${ }^{5}$, Orji Oliver Chukwuma ${ }^{6}$, \\ Nwachukwu Daniel ${ }^{7}$, Eze Anthonius Anayochukwu ${ }^{8}$ \\ ${ }^{1,8}$ Senior Lecturer, ${ }^{5} \mathrm{PhD}$ Student, Departments of Medical Biochemistry, ${ }^{7}$ Senior Lecturer, Department of Medical \\ Physiology, College of Medicine, University of Nigeria, Enugu Campus, Nigeria. ${ }^{2}$ Student, ${ }^{3}$ Medical Laboratory Scientist, \\ ${ }^{4}$ Senior Lecturer, ${ }^{6}$ Assistant Lecturer, Department of Medical Laboratory Science, Faculty of Health Science and \\ Technology, University of Nigeria, Enugu Campus, Nigeria
}

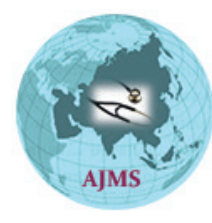

A B S T R A C T

Background: Normal metabolic functions generate highly reactive compounds called free radicals in the body. Exercise is accompanied by increased oxygen consumption and enhanced Reactive Oxygen Species (ROS)/free radical production in humans. These molecules react with cellular molecules such as proteins, lipids and carbohydrates, and denature them. As a result of this, vital cellular structures and functions are lost and ultimately resulting in various pathological conditions. Aims and Objective: The objective of this study was to determine the effect of physical exercise on lipid peroxidation and antioxidant enzymes in apparently healthy individuals. Materials and Methods: A total of 96 samples were collected from apparently healthy adults aged between 18 and 25 years before and after morning exercise session. The samples were analyzed for Total Antioxidant Activity (TAS), pro-oxidant biomarker- malondialdehyde (MDA) and antioxidant enzymes - catalase (CAT) and glutathione peroxidase (GPx) by spectrophotometic method. Results: The result shows that TAS after exercise was significantly higher $(p<0.001)$ than the level before exercise. The MDA level of the subjects after exercise was equally statistically significantly $(p<0.001)$ higher than the level before exercise. This study therefore shows that aerobic exercise increases pro-oxidant markers and possibly induces oxidative stress, the effect of which is countered by an increase in TAS, CAT and GPx. Conclusion: Oxidative stress following exercise may not deter the benefits of physical exercise.

Key words: MDA, TAS, CAT, GPx, oxidative stress

\section{Access this article online}

Website:

http://nepjol.info/index.php/AJMS DOI: 10.3126/ajms.v7i6.15245

E-ISSN: 2091-0576 P-ISSN: $2467-9100$

\section{INTRODUCTION}

The ability of the cell to utilize oxygen has provided humans with the benefit of metabolizing fats, proteins, and carbohydrates for energy. Oxygen is a highly reactive molecule that is capable of becoming part of potentially damaging molecules commonly called free radicals or reactive oxygen species. About $5 \%$ or more of the inhaled oxygen is converted to ROS such as superoxide, hydrogen peroxide and hydroxyl radicals by univalent reduction of oxygen. ${ }^{1}$ Thus cells under aerobic condition are always threatened with the insult of ROS, which however are efficiently taken care of by the highly powerful antioxidant systems of the cell. ${ }^{1}$ The enzymatic and non-enzymatic antioxidant defenses include superoxide dismutase (SOD), glutathione peroxidase (GPX), catalase (CAT), ascorbic acid (vitamin C), $\alpha$-tocopherol (vitamin E), glutathione (GSH), $\beta$-carotene, and vitamin A..$^{2-4}$ Whenever the balance between ROS production and antioxidant defense is lost, 'oxidative stress' results which through a series of events deregulates the cellular functions leading to various pathological conditions. ${ }^{5,6}$

Aerobic exercise is accompanied by increased oxygen consumption which in turn may increase ROS production. 
Many studies suggest that such physical activity enhanced ROS production both in animals and in humans. ${ }^{7}$ However, this phenomenon cannot occur with low exercise intensity. In such a case, antioxidant capacity is not overwhelmed and ROS-induced damage does not appear. Moreover, the more intense the exercise is, the more important the ROS production and the oxidative stress are. ${ }^{8}$ The increase of free radical production specific for the anaerobic phase of exercise may be mediated through various pathways in addition to electron leakage which is thought to be the main source of ROS due to aerobic exercise. ${ }^{9,10}$ Ischemic reperfusion of the active muscle is greatly involved in oxidative stress during and after the anaerobic phase of exercise. This type of exercise significantly enhances the catabolism of purins and provokes a fast deoxygenation which increases the activity of xanthine oxidase and accelerates free radical production. Therefore, this study was carried out to measure the effect of exercise on the activity of antioxidant enzymes in erythrocytes, and MDA level and total antioxidant concentration in plasma in sedentary young men.

\section{MATERIAL AND METHODS}

\section{Subjects}

Ninety six (96) apparently healthy non smoking male and female subjects aged between 18 and 30 years were recruited for this study from Enugu State University Teaching hospital, Parklane, Enugu. Informed consent was obtained from all the participants after 30 minutes talk show on exercise and its health benefits. The criteria for inclusion were established as follows: age bracket of 18-30 years, consent to participate in all stages of data collection, no history of injury in the one month prior to the study. The exclusion criteria includes: self reported history of myocardial infarction, cardio-pulmonary disease, or any organ dysfunction or those on any form of medication.

\section{Blood collection}

Blood samples were collected from the subjects before and after a bout of exercise performed between 6:30 am and 8:30 am and before any breakfast after an overnight fast. $10 \mathrm{mls}$ of blood was drawn from each subject from the anticubital vein before exercise and immediately after exercise into plain sterile test tube. The clotted samples were centrifuged to extract the serum which was stored at $-20^{\circ} \mathrm{C}$.

\section{Biochemical analysis}

Malondiadehyde (MDA), a measure of lipid peroxidation, was analyzed using the method of Ohkawa et al., 1979. ${ }^{11}$ In this method $2.5 \mathrm{ml}$ of $10 \%$ trichloroacetic acid and
$0.5 \mathrm{ml}$ of plasma were mixed in a tube and incubated for $15 \mathrm{~min}$ at $90^{\circ} \mathrm{C}$ before cooling with cold water. The mixture was subsequently centrifuged at $3000 \mathrm{rpm}$ for $10 \mathrm{~min}$. Thereafter, $2 \mathrm{ml}$ of the supernatant was added to $1 \mathrm{ml}$ of $0.675 \%$ TBA solution in a test tube. The tube was sealed and incubated at $90^{\circ} \mathrm{C}$ for $15 \mathrm{~min}$ and then cooled to room temperature. The optical density was measured at $532 \mathrm{~nm}$ by a spectrophotometer. MDA was expressed as $\mathrm{nmol} / \mathrm{ml}$.

Total antioxidant status (TAS) was assessed according to the method of Koracevic et al., 2001. ${ }^{12}$ It was based on the principle that a standardized solution of Fe-EDTA complex reacted with hydrogen peroxide by a Fenton-type reaction, leading to the formation of hydroxyl radicals. These reactive oxygen species degraded benzoate, resulting in the release of TBARS. Antioxidants from the added human serum caused suppression of the production of TBARS that was proportional to their concentration. This reaction was measured spectrophotometrically at $532 \mathrm{~nm}$ and the inhibition of colour development was used as a measure of the TAS.

Catalase activity was measured following the method of Aebi, 1984. ${ }^{13}$ Briefly, the activity was determined by measuring the decrease in absorbance at $240 \mathrm{~nm}$ of a reaction mixture consisting of $\mathrm{H}_{2} \mathrm{O}_{2}$, in phosphate buffer, $\mathrm{pH} 7.0$ and requisite volume of serum sample. The molar extinction coefficient of $43.6 \mathrm{M} \mathrm{cm}^{-1}$ was used to determine catalase activity. The specific activity was calculated and was expressed as $\mu$ moles $/ \mathrm{min} / \mathrm{mg}$ of total protein. Glutathione Peroxidase activity was determined using the Flohe and Gunzler method of $1984 .{ }^{14}$

\section{Ethics}

The procedures followed in this study were in accordance with the ethical standards of University of Nigeria ethics committee on human experimentation.

\section{Statistical analysis}

Results were expressed as mean \pm standard deviation (SD). For the comparison, unpaired Student t-test was used.

\section{RESULTS}

The serum pro-oxidant biomarker - malondialdehyde (MDA) of adolescent volunteers after an aerobic exercise was elevated (1.78 \pm 0.2$)$ with percentage increment of $147 \%$ compared with the basal level $(0.72 \pm 0.02)$. The $p$ value of $<0.05$ showed a statistically significant difference between the post-exercise and per-exercise values (Figure 1).

Catalase activity in serum of the adolescent volunteers was also elevated after exercise $(0.313 \pm 0.01)$ with percentage increment of $20.4 \%$ relative to the basal level of $0.26 \pm 0.01$ as shown in Figure 2. 
Glutathione peroxidase activity after physical exercise showed a slight increase $(0.74 \pm 0.02)$ with percentage increment of $19.4 \%$ compared with the basal level $(0.62 \pm 0.02)$ as shown in Figure 3 .

The mean TAS of $77.31 \pm 3.675$ after exercise was statistically significantly higher $(p<0.001)$ than the mean TAS of $22.20 \pm 2.486$ before exercise (Figure 4).

\section{DISCUSSION}

While regular exercise is associated with numerous health benefit, it can be viewed as an intense physical stressor leading to increased oxidative cellular damage, likely due to enhanced production of ROS. ${ }^{15} \mathrm{~A}$ single bout of exercise can result in activation of several distinct systems of radical generation. Many studies have reported that acute aerobic exercise contributes to oxidative stress, especially when performed at high intensity levels and this can be linked to

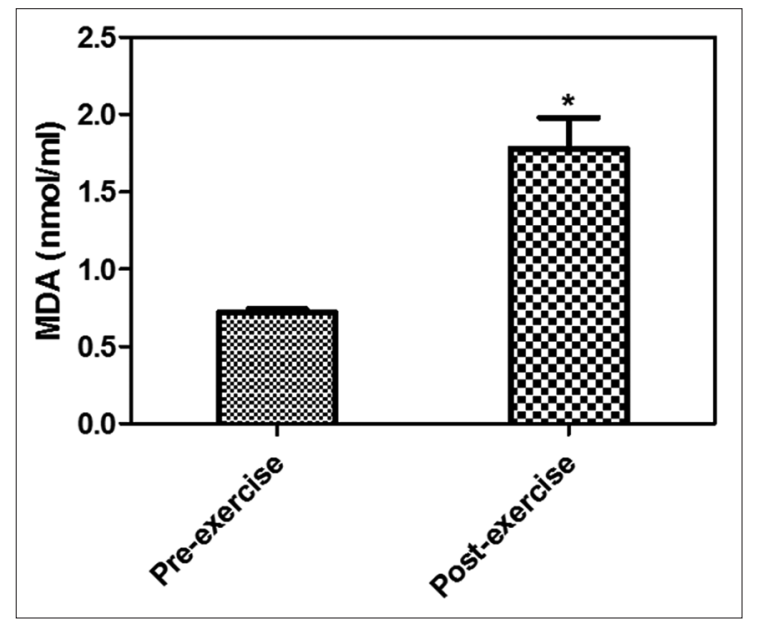

Figure 1: Malonaldehyde (MDA) level in the serum of subjects before and after an exercise session. ${ }^{*} \mathrm{P}<0.05$ (unpaired Student's t-test)

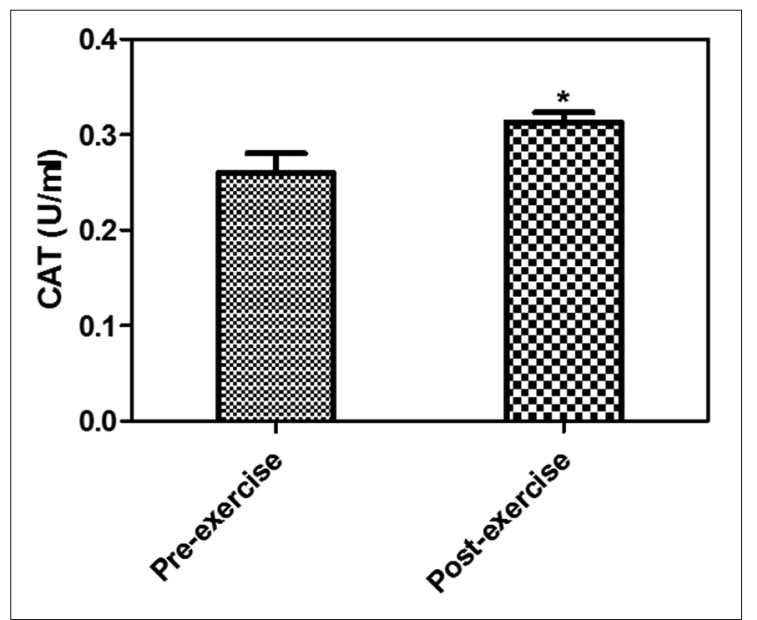

Figure 2: Catalase (CAT) level in the serum of subjects before and after an exercise session. ${ }^{*} \mathrm{P}<0.05$ (unpaired Student's t-test) increased pro-oxidant activity and inadequate antioxidant activity relative to pro - oxidants. ${ }^{16}$

Only exercise of sufficient intensity or duration appears to lead to increase in free radical production to overwhelm the antioxidant defenses. ${ }^{17-19}$ As the results have shown, there is a significant increase in the serum malondialdehyde, a marker of lipid peroxidation due to oxidative stress in the volunteers after an intensive bout of physical exercise. This agrees with the findings made by Miyazaki et al., $2001^{20}$ in which they stated that a single bout of exercise increased blood level of MDA. Other studies by Balci et al., 201021 and Branth et al., $2009^{22}$ also showed that MDA increased in untrained individuals doing acute exercise in unusual (high) intensity and the rise in serum MDA levels is dependent on exercise intensity.

The serum levels of catalase activity in the adolescent volunteers were elevated after exercise than the basal level.

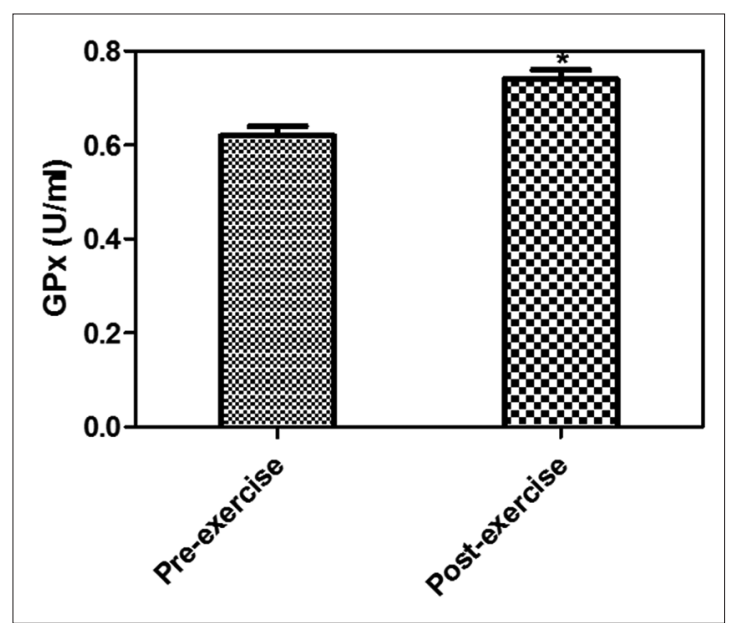

Figure 3: Glutathione peroxidase (GPx) level in the serum of subjects before and after an exercise session. ${ }^{*} \mathrm{P}<0.05$ (unpaired Student's t-test)

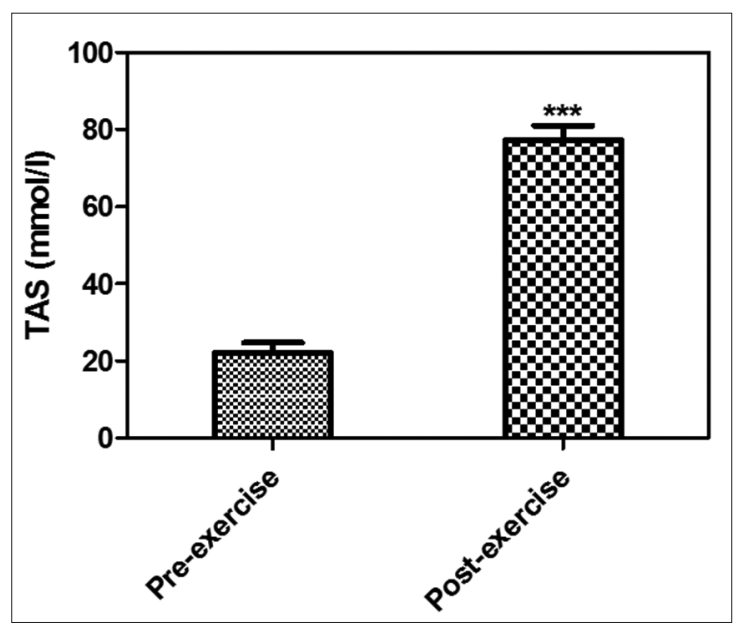

Figure 4: TAS level in the serum of subjects before and after an exercise session. ${ }^{* \star *} \mathrm{p}<0.001$ (unpaired Student's t-test) 
This is in line with the work by Tian et al., 2010, ${ }^{23}$ where they investigated the oxidant and antioxidant status before and after exercise in adolescent runners and found that catalase level increased $2 \mathrm{hrs}$ after the run.

Glutathione peroxidase (GPx) activity showed an elevated value from the basal to the post-exercise value by $16.2 \%$ and this increase was found to be significant. In this study, both the catalase (CAT) and glutathione peroxidase (GPx) activities were raised from their basal values after physical exercise. This work also supports the findings of Vider et al, $2007^{24}$ where they reported that CAT increased after exercise at maximal intensity. It is also in support of the studies conducted by Cebrian et al, $2006^{25}$ which demonstrated that CAT and GPx activities were raised after exercise than at rest. The increased CAT and GPx activities during exercise provide not only protection from ROS but also attenuate aging process and promote health.

\section{CONCLUSION}

This study shows that oxidative stress following exercise do exist, but may not deter the benefits of physical exercise.

\section{REFERENCES}

1. Praveen $\mathrm{K}$ and Ashish W. Antioxidant Enzymes and Human Health, Antioxidant Enzyme, Prof. Mohammed Amr El-Missiry (Ed.), 2012.

2. Beaudeux JL, Gardes-Albert M, Delattre J, Legrand A, Rousselet F and Peynet J. Resistance of lipoprotein (a) to lipid peroxidation induced by oxygenated free radicals produced by gamma radiolysis: a comparison with low-density lipoprotein. Biochem J 1996; 314: 277-284.

3. Hall L, Williams K, Perry ACF, Frayne J and Jury JA. The majority of human glutathione peroxidase type 5 (GPX5) transcripts are incorrectly spliced: implications for the role of GPX5 in the male reproductive tract. Biochem J 1998; 333: 5-9.

4. Jankowska R, Passowicz-Muszynska E, Banas $T$, Marcinkowska A and Medrala W. The influence of vitamin A on production of oxygen free radicals and activity of granulocyte catalase in patients with chronic bronchitis. Pneumonol Alergol Pol 1994; 62: 628-633.

5. Uday B, Dipak D and Ranajit KB. Reactive Oxygen Species: oxidative damage and pathogenesis. Curr Sci 1999; 77: 658-666.

6. Chitra KP and Pillai KS. Antioxidants in Health. Ind J Physiol Pharmacol 2002; 46 (1): 01-05.

7. Mastaloudis A, Leonard SW and Traber MG. Oxidative Stress in Athletes during Extreme Endurance Exercise. Free Radical Biology \& Medicine 2001; 31 (7): 911-922.

8. Vladimir LJ, Jakovljevic DC, Vladimir Z, Dusica D and Dragan D.
Exercise and Oxidative Stress, Oxidative Stress - Environmental Induction and Dietary Antioxidants, Dr. Volodymyr Lushchak (Ed.), 2012.

9. Deaton $\mathrm{CM}$ and Marlin DJ. Exercise associated oxidative stress. Clin Tech Equine Prac 2003; 2(3): 278-291.

10. Groussard C, Rannou-Bekono F, Machefer G, Chevanne M, Vincent S, Sergent O, et al. Changes in Blood Lipid Peroxidation Markers and Antioxidants after a Single Sprint Anaerobic Exercise. European Journal of Applied Physiology 2003; 89 (1): 14-20.

11. Ohkawa $\mathrm{H}$, Ohishi $\mathrm{N}$ and Yaji $\mathrm{K}$. Assay for lipid peroxides in animal tissues by thiobarbituric acid reaction. Anal Biochem 1979; 95: 351-356.

12. Koracevic D, Koracevic G, Djordjevic V, Andrejevic $S$ and Cosic V. Method for the measurement of antioxidant activity in human fluids. J Clin Pathol 2001; 54: 356-356.

13. Aebi H. In: Catalase in Vitro. Methods in Enzymology. Colowick SP, Kaplan NO. Acad. Press; 1984; 105; 114-121.

14. Flohe $L$ and Gunzler WA. Assays of glutathione peroxidase. Methods in Enzymol 1984; 105: 114-121.

15. Bloomer RJ, Goldfarb AH, Wideman L, Mc Kenzie MJ and Consitt LA. Effects of acute aerobic and anaerobic exercise on blood markers of oxidative stress. J Strength Cond Res 2005; 19 (2): 276-285.

16. Alessio HM, Hagerman AE, Fulkerson BK, Ambrose J, Rice RE and Wiley RL. Generation of reactive oxygen species after exhaustive aerobic and isometric exercise. Med Sci Sports Exerc 2000; 32 (9): 1576-1581.

17. Niess AM, Hartmann A, Grunert-Fuchs M, Poch B and Speit G. DNA damage after exhaustive treadmill running in trained and untrained men. Int J Sports Med 1996; 17 (6): 397-403.

18. Quindry JC, Stone WL, King J and Broeder CE. The effects of acute exercise on neutrophils and plasma oxidative stress. Med Sci Sports Exerc 2003; 35 (7): 1139-1145.

19. Poulsen HE, Loft $S$ and Vistisen K. Extreme exercise and oxidative DNA modification. J Sports Sci 1996; 14 (4): 343-346.

20. Miyazaki H, Ookawaa T, Kizaki $T$ and Haga S. Strenuous endurance training in human reduces oxidative stress following exhaustive exercise. Eur J Appl Physiol 2001; 84:1-6.

21. Balci SS, Okudan N, Pepe $\mathrm{H}$ and Gokbeb $\mathrm{H}$. Changes in lipid peroxidation and antioxidant capacity during walking and running of the same and different intensities. J Strenght Cond Res 2010; 24: 2545-2550.

22. Branth S, Hambreus L, Piehi-Aulin K, Olsson $R$ and Stridsberg M. Metabolic stress like condition can be induced by prolonged strenuous exercise in athletes. UPSala $\mathrm{J}$ Med 2009; 114: 12-25.

23. Tian Y, Nie J, Tong TK, Baker JS, Thomas NE and Shi Q. Serum Oxidants and antioxidant status during early and late recovery periods following an all-out 21-km run in trained adolescent runners. Eur J Appl Physiol 2010; 110: 971-976.

24. Vider J, Lehtmaa J, Kulisaar T, Vihalemm T and Zimer K. Acute immune response in respect to exercise-induced oxidative stress. Pathiophysiology 2007; 7: 263-270.

25. Cebrain I, Gomez-Trullen E, Reiter RJ and Garcia JJ. Effects of acute exercise on lung antioxidant enzymes in young and old rats. Mechanisms of Aging and Development 2006; 127 (4): 384-390.

Authors Contribution:

IJE, IAJ, ECN, OIS, OOC - Conception, design, data collection, laboratory analysis, literature search and critical revision of the manuscript;

OKC, ND, EAA - Data collection, literature search/review, statistical analysis/interpretation, preparation of the manuscript and critical revision of the manuscript.

Source of Support: Nil. Conflict of Interest: None. 